\title{
Does Depression Damage Health?
}

\section{John Wattis}

Shah, A., Phongsathorn, V., George, C., Bielawska, C. and Katona, C. L. E. Does psychiatric morbidity predict mortality in continuing care geriatric inpatients? International Journal of Geriatric Psychiatry 8 (I993), $255^{-259}$.

This I 990 study screened all patients in continuing care geriatric beds in a London Health District for psychiatric morbidity and physical dependency. The sample was followed up after one year. Nearly half the patients were dead at follow up - a higher mortality than in private nursing home beds, probably due to a more severely ill/disabled population. Dementia, physical dependency and depression, as measured by the depressive signs score (DSS), were associated with mortality but after further analysis to partial out independent effects, the DSS was the only significant predictor of mortality.

\section{COMMENT}

This was a limited study. More than $80 \%$ of the subjects were demented, reflecting the high prevalence of dementia in many continuing-care settings. Depression was measured by the DSS, an instrument designed especially to measure depression in demented people; its association with higher mortality rates was therefore of interest. The authors cite earlier studies which have shown that depression is poorly recognised and poorly treated in institutions, and they suggest that the DSS could be used to recognise patients at risk of early death, allowing treatment interventions to be assessed for their potential in improving the quality as well as the duration of life.

Dewey, M. R., Davidson, I. A. and Copeland, J. R. M. Expressed wish-to-die and mortality in older people: a community replication. Age and Ageing, 22 (1993), 109-1 I 3.

The three-year mortality of $\mathrm{I}, 06_{3}$ community residents aged $6_{5}$ and over was assessed using the Geriatric Mental State schedule (GMS) and analysed to examine the effect of specific depressive symptoms and physical illness. Expressed 'wish-to-die' was confirmed as a predictor of mortality in depressed patients controlling for age, sex and cognitive impairment. The suggestion that the effect of depressive symptoms on mortality might be a masked effect of physical illness was not confirmed. 
This paper is part of the findings from a wide-ranging study conducted by Copeland and his colleagues in Liverpool. The authors conclude that it demonstrates a link between depressed mood and mortality which becomes stronger when more specific symptoms are considered. The indicator of physical ill health was a crude one, being based on physical medication; but nevertheless it predicted a two-fold increase in mortality. However, when physical illness was controlled, the relationship between indicators of depression and mortality remained although it was weaker.

Old age depression interest group (OADIG): How long should the elderly take anti-depressants? A double-blind placebo-controlled study of continuation/prophylaxis therapy with dothiepin. British Joumal of Psychiatry, I62 (1993), I 75-182.

This paper reports a multi-centre trial of continuation/prophylaxis therapy with dothiepin in 69 recovered patients with RDC major depressive disorder over two years. Treatment reduced the relative risk of relapse by 2.5. Past but not current physical illness was also associated with a favourable prognosis whereas a prolonged index illness trebled the relative risk of relapse. The authors conclude that elderly people who recover from a major depressive illness should continue treatment for at least two years and possibly indefinitely.

\section{COMMENT}

At last we have a plausibly authoritative study of how long we should continue to treat patients with a research diagnosis of major depressive disorder (certainly a different group from those described in the first abstracted study and probably a different group from those described in the second). The clinical answer, as many have suspected, is for a long time. Other anti-depressants, with different side effects and less toxicity in overdose, may now be preferred over dothiepin but it seems clear that prolonged treatment substantially reduces the risk of relapse, which directly benefits the patient and indirectly assists a health service by reducing the need for re-admission. 
McMurdo, M. E. and Rennie, L. A controlled trial of exercise by residents of old people's homes. Age and Ageing, 22 (1993), I 1-15.

This study reports a controlled trial of seated exercise among residents of United Kingdom local authority homes for elderly people. Fortynine residents aged 64-9 I years volunteered for this seven-month project and participated in either twice-weekly exercise or reminiscence sessions. Primary outcome measures were postural sway, flexibility of the spine and knees, hand-grip strength and functional capacity. The average attendance at the sessions was over $91 \%$ for exercise and $86 \%$ for reminiscence. The exercise group improved more not only on physical measures but also on self-rated depression as measured by the geriatric depression scale (GDS).

\section{COMMEN T}

Unfortunately the psychiatric aspects of this study are not reported in detail and although the small improvement in cognitive function was not statistically significant, we are not told how many of the volunteers suffered from dementia or depressive disorder or how representative the volunteers were of the overall population. However, in both groups the improvement in the GDS scores was significantly greater in the exercise group.

These four disparate papers each have a bearing on the issue of depression in old age. The Royal College of Psychiatrists as one of its themes for the European Year of the Elderly is promoting attention to 'Depression: underdiagnosed and under-treated'. The first two studies demonstrate that depression (albeit defined in different ways) affects mortality and not just the quality of life. The third study stresses the importance of the adequate treatment of psychiatrically-diagnosed severe depressive illness, while the last suggests that, at least for those with minor degrees of mood disturbance, even a limited programme of exercise may improve mood. Collectively they provide innumerable leads to pursue. One of the major problems from the psychiatrist's point of view, however, is that depression in old age is often seen as a normal state and not as a disorder which merits prevention and intervention. If only we could invent some eponym equivalent to 'Alzheimer's Disease'; perhaps we would then attract to this essentially treatable and perhaps preventable group of disorders the public attention it deserves.

The Newsam Centre,

Seacroft Hospital, Leeds 